\title{
Online Over Time Scheduling on Parallel-Batch Machines: A Survey
}

\author{
Ji Tian $\cdot$ Ruyan Fu $\cdot$ Jinjiang Yuan
}

Received: 30 October 2014/Revised: 3 November 2014/ Accepted: 4 November 2014/

Published online: 26 November 2014

(C) Operations Research Society of China, Periodicals Agency of Shanghai University, and SpringerVerlag Berlin Heidelberg 2014

\begin{abstract}
Online scheduling is a rapidly developed branch in scheduling theory. In this paper, we present an extensive survey for online over time scheduling on parallel-batch machines. Some open problems are proposed for further research.
\end{abstract}

Keywords Online scheduling Parallel-batch machines - Competitive ratio

\section{Introduction}

In parallel-batch scheduling, we have $n$ jobs $J_{1}, \cdots, J_{n}$ and $m$ parallel-batch machines $M_{1}, \cdots, M_{m}$. Each job $J_{j}$ has a release date $r_{j} \geqslant 0$, a processing time $p_{j}>0$, a weight $w_{j}>0$, and a delivery time $q_{j} \geqslant 0$. Each parallel-batch machine $M_{i}$ can process up to $b$ jobs simultaneously as a batch. Here, a batch is a subset of jobs and $b$ is the capacity of the batches. If $b=\infty$, the model is called unbounded batching. Alternatively, if $b<\infty$, the model is called bounded batching. The processing time of a batch is defined as the maximum processing time of the jobs in the batch. A batch can be started to processing at a time $t$ if all the jobs in the batch are released by time $t$. Furthermore, in a schedule $\pi$, the jobs in a batch have the same starting time and the same completion time, respectively. Then a schedule for

This work was supported by National Natural Science Foundation of China (NSFC, No. 11301528) and NSF of Jiangsu Province (No. BK20130169). Fu was also supported by NSFC (No. 11201439), and Yuan was also supported by NSFC (Nos. 11271338 and 11171313).

J. Tian · R. Fu

College of Sciences, China University of Mining and Technology, Xuzhou 221116, Jiangsu, China

J. Yuan $(\bowtie)$

School of Mathematics and Statistics, Zhengzhou University, Zhengzhou 450001, China

e-mail: yuanjj@zzu.edu.cn 
the parallel-batch scheduling can be determined by partitioning the jobs into batches and assigning the batches to the available time spaces of the $m$ parallel-batch machines without overlap.

Parallel-batch scheduling was motivated by semiconductor manufacturing. Uzsoy et al. [45, 46], Avramidis et al. [2], and Mathirajan and Sivakumar [31] described the application in the semiconductor manufacturing process in detail. The fundamental model of bounded parallel-batch scheduling was first introduced by Lee et al. [21]. For problem $1 \mid p-$ batch,$b<\infty \mid C_{\max }$, it was reported by Lee and Uzsoy [22] that the optimal schedule is given by the full batch longest processing time (FBLPT) rule proposed first by Bartholdi. An extensive discussion of the unbounded parallelbatch scheduling problem was provided by Brucker et al. [4]. Recent developments on this topic can be found in [5] and [6]. With dynamic job arrivals and the capacity $b$ being infinite, Lee and Uzsoy [22] presented a dynamic programming algorithm to solve problem $1 \mid p$-batch, $b=\infty, r_{j} \mid C_{\max }$ in $O\left(n^{2}\right)$ time. For the same problem, Poon and Zhang [34] presented an improved $O(n \log n)$-time algorithm. For the bounded parallel-batch scheduling problem $1 \mid p$-batch, $b<\infty, r_{j} \mid C_{\max }$, Liu and Yu [26] showed that the problem with only two arrival times is NP-hard, and gave a pseudo-polynomial-time algorithm in case of fixed number of arrival times. Brucker et al. [4] proved that the general problem is NP-hard in the strong sense.

Online scheduling is a relatively new topic in scheduling research and has been extensively studied in the last decade. While there are different meanings of online scheduling, the term "online" in this paper means that jobs arrive over time.

In the online over time environment, jobs arrive over time and we do not have any information about the jobs in advance. The information of each job $J_{j}$ can be known only at the arrival time $r_{j}$ of the job. Hence, we must schedule the available jobs without information of the future jobs. Usually, the information of a job $J_{j}$ includes its release time $r_{j}$, its processing time $p_{j}$, its delivery time $q_{j}$, its weight $w_{j}$, its job family, etc. In the makespan minimization, the necessary information of a job is its release time $r_{j}$, its processing time $p_{j}$, and its job family (when the jobs are partitioned into incompatible job families).

The quality of an online algorithm is measured by its competitive ratio. Suppose that we are considering an online scheduling problem $\mathcal{P}$ to minimize a certain objective function. Let $C_{\mathrm{on}}(L)$ and $C_{\mathrm{opt}}(L)$ denote, respectively, the objective value of an online algorithm $H$ and the off-line optimal value for an input $L$. We say that algorithm $H$ is $R$-competitive for some $R \geqslant 1$ if $C_{\text {on }}(L) / C_{\text {opt }}(L) \leqslant R$ for all inputs $L$. The competitive ratio $R_{H}$ of algorithm $H$ is defined as

$$
R_{H}=\sup _{L}\left\{C_{\text {on }}(L) / C_{\text {opt }}(L)\right\} \text {. }
$$

In this case, we also say that algorithm $H$ is $R_{H}$-competitive, and $R_{H}$ is an upper bound of the competitive ratio of problem $\mathcal{P}$.

Given an online scheduling problem $\mathcal{P}$, we say that it has a lower bound $\rho$, if every online algorithm for $\mathcal{P}$ has a competitive ratio at least $\rho$. Furthermore, if $\mathcal{A}$ is an online algorithm for $\mathcal{P}$ such that the competitive ratio of $\mathcal{A}$ is exactly the lower bound $\rho$ for $\mathcal{P}$, we say that $\mathcal{A}$ is the best possible online algorithm for $\mathcal{P}$. 
Some examples of the studies on online scheduling problems (with jobs arriving over time) can be found in [1, 9, 18, 19], and [51], among many others.

In general, the competitive ratio of an online algorithm may be improved if some information of the jobs is known in advance. This scenario is described as "semionline" in the literature. There have been plenty researches concerning semi-online scheduling with jobs arriving over list. For example, Cheng et al. [8] studied the semi-online scheduling on parallel machines with given total processing time. Seiden et al. [37] studied the semi-online scheduling on parallel machines with decreasing job sizes. Tan and He [38] studied semi-online scheduling on two parallel machines with combined partial information. In contrast, there are only a few researches concerning semi-online scheduling with jobs arriving over time. The representative publication was given by Hall et al. [17]. They studied the semionline scheduling on a single machine to minimize the sum of weighted completion time with known arrival times of the jobs.

In this survey, we report the developments on online scheduling of parallel-batch machines. An informal version of this paper [47] was presented in the web site http://www.paper.edu.cn.

Let $f$ be the objective function to be minimized. Using the standard scheduling classification scheme of Lawler et al. [20], the scheduling models considered in this survey can be summarized as

$$
P \mid \text { online, } p \text {-batch, } b, \beta \mid f \text {, }
$$

where $b<n$ or $b=\infty, \beta \in\left\{\right.$ restart,L-restart, f families, Agreeable $\left(p_{j}, q_{j}\right), q_{j} \geqslant$ $\left.p_{j}, q_{j} \leqslant p_{j}, J^{*}(t), p^{*}(t), r^{*}(t)\right\}$ and $f \in\left\{C_{\max }, D_{\max }, F_{\max }, \sum C_{j}, \sum w_{j} C_{j}\right\}$.

The notations appearing in the above models are described as follows:

- $C_{j}$ is the completion time of job $J_{j}$.

- $C_{\max }=\max \left\{C_{j}: 1 \leqslant j \leqslant n\right\}$ is the maximum completion time of all jobs.

- $D_{\max }=\max \left\{C_{j}+q_{j}: 1 \leqslant j \leqslant n\right\}$ is the maximum delivery time by which all jobs have been delivered.

- $F_{\max }=\max \left\{C_{j}-r_{j}: 1 \leqslant j \leqslant n\right\}$ is the maximum flow time of all jobs.

- $\sum_{1 \leqslant j \leqslant n}\left(w_{j}\right) C_{j}$ is the total (weighted) completion time of jobs.

- "Restart" means that a running task may be interrupted, losing all the work done on it. In this case, an interrupted job becomes unscheduled. Allowing restart reduces the impact of a wrong decision.

- "L-restart" means that batches are only allowed limited restarts. If a batch has been restarted one time, then all jobs in it are considered as interrupted jobs. Any new batch that contains interrupted jobs cannot be restarted any more. That is, any job is allowed to restart only once.

- " $f$ families" means that the jobs are partitioned into $f$ incompatible families so that the jobs in different families cannot be processed in a common batch.

- "Agreeable $\left(p_{j}, q_{j}\right)$ " means that for jobs $J_{i}$ and $J_{j}$, if $p_{i} \geqslant p_{j}$, then $q_{i} \geqslant q_{j}$.

- " $q_{j} \leqslant p_{j}$ " means that, for each job $J_{j}$, the processing time $p_{j}$ is no less than the delivery time $q_{j}$. 
- " $q_{j} \geqslant p_{j}$ " means that, for each job $J_{j}$, the processing time is no longer than the delivery time $q_{j}$.

- " $J^{*}(t)$ " means that at time $t$ the information of the first longest job arriving after time $t$ is given.

- " $p^{*}(t)$ " means that at time $t$ the processing time of the first longest job arriving after time $t$ is given.

- " $r^{*}(t)$ " means that at time $t$ the arrival time of the first longest job arriving after time $t$ is given.

\section{To Minimize Makespan $C_{\max }$}

Many researchers focused on studying online scheduling on parallel-batch machines to minimize makespan, and they provided so many distinct online algorithms, even for the same problem. In fact, online algorithms could be divided into two classes. One is called delay algorithm, which means that the algorithm always waits until the time moment satisfying some conditions, even the jobs are available and some machines are idle. The other one is called greedy algorithm which always immediately assigns available jobs as long as there exist idle machines, with no waiting time.

Zhang et al. [51] and Deng et al. [9] presented basic ideas for the research of online scheduling on parallel-batch machines, which have been widely accepted in the onward research. They investigated online scheduling on a single batch machine to minimize makespan. For unbounded version, they independently provided the same best possible online delay algorithm with a competitive ratio of $\frac{\sqrt{5}+1}{2}$. Later, Poon and $\mathrm{Yu}$ [35] further provided a best flexible parameterized online algorithm, which contains the above algorithm. For the bounded case, Lee and Uzsoy [22] presented a greedy algorithm, which was proved to be 2-competitive by Liu and Yu [26]. Zhang et al. [51] proposed two algorithms based on FBLPT rule with competitive ratio 2, while they proved that no online algorithm has a competitive ratio less than $\frac{\sqrt{5}+1}{2}$. Poon and $\mathrm{Yu}$ [36] presented a class of FBLPT (full batch longest processing time)-based algorithms including the above three algorithms with competitive ratio 2 . For the special case $b=2$, they provided an online algorithm with a competitive ratio of $7 / 4$.

Fu et al. [14] investigated the unbounded model with restarts. They proved that no online algorithm has a competitive ratio less than $\frac{5-\sqrt{5}}{2}$, and they presented a $\frac{3}{2}$ competitive algorithm. Later, Yuan et al. [49] designed a best possible online algorithm with a competitive ratio $\frac{5-\sqrt{5}}{2}$. Fu et al. [15] studied the same problem with limited restarts, in which a job cannot be restarted twice. They proved that the best online algorithm is $\frac{3}{2}$-competitive. With restarts, these results improve the known $\frac{\sqrt{5}+1}{2}$-competitive. For the bounded model with restarts or limited restarts, Liu and Yuan [29] studied the special case that all jobs have equal processing time. They proposed two best possible algorithms with four different competitive ratios depending on the capacity $b$ of a batch.

There are several results about the online scheduling problem on a single batch machine with job families but only the unbounded model was investigated. When 
the number of families is 2, Fu et al. [16] proposed a best possible online algorithm with competitive ratio $\frac{\sqrt{17}+3}{4}$. For the general version with $f$ families, Nong et al. [32] firstly provided an online algorithm with competitive ratio 2 and proved that the algorithm is best possible when $f$ tends to infinity. Lastly, when the number $f$ of job families is given in advance, Fu et al. [13] provided a best possible online algorithm with a competitive ratio of $1+\frac{\sqrt{4 f^{2}+1}-1}{2 f}$, which extends the special case $f=1$ including the results for unbounded case in Zhang et al. [51], Deng et al. [9], and Poon and $\mathrm{Yu}$ [35].

For semi-online scheduling on a single unbounded batch machine to minimize makespan, given the information " $J^{*}(t)$ " (the first longest job) or " $p^{*}(t)$ " (the processing time of the first longest job) arriving after time $t$, Yuan et al. [50] proposed best possible online algorithms with competitive ratio $\frac{5-\sqrt{5}}{2}$. The result implies that the information " $p^{*}(t)$ " dominates " $r^{*}(t)$ " since the information " $J^{*}(t)$ " consists of " $p^{*}(t)$ " and " $r^{*}(t)$ " which is the arrival time of the first longest job arriving after time $t$. Given the information " $r^{*}(t)$ ", Yuan et al. [50] provided a $\frac{3}{2}$-competitive algorithm and showed a lower bound 1.442 .

Online scheduling on $m$ parallel-batch machines was extensively studied as well. For the unbounded version, Zhang et al. [51] provided a lower bound $\sqrt[m+1]{2}$ of competitive ratio and presented an online algorithm $P H\left(\theta_{m}\right)$ with a competitive ratio of $1+\theta_{m}$, where $0<\theta_{m}<1$ and $\theta_{m}=\left(1-\theta_{m}\right)^{m-1}$. In the case that jobs have identical processing time, they proposed two best possible online algorithms with competitive ratios of $1+\beta_{m}$, where $(1+\beta)^{m+1}=\beta+2$, for $b=\infty$ and $\frac{\sqrt{5}+1}{2}$ for $b<\infty$. When $m=2$, Nong et al. [33] presented an online algorithm with competitive ratio $\sqrt{2}$. Later, Tian et al. [41] proved that the lower bound of this problem is $\sqrt{2}$ and they designed a distinct best possible algorithm. For the general version with $m$ being arbitrary, Tian et al. [40] and Liu et al. [27] independently presented two distinct best possible algorithms with a competitive ratio of $1+\gamma_{m}$, where $\gamma_{m}=\frac{\sqrt{m^{2}+4}-m}{2}$. Tian et al. [40] also provided a best possible $\frac{3}{2}$-competitive dense algorithm that always immediately processes the available jobs as a batch on one idle machine when there are at least two idle machines. Their result generalized the work of Nong et al. [33] and Tian et al. [41] for $m=2$. Tian et al. [42] also considered the same problem with incompatible families, where the number of families is equal to the number of machines $m$. They first gave a lower bound $\frac{\sqrt{5}+1}{2}$ on the competitive ratio of any online algorithm, then they provided an online algorithm $H_{m}(\theta)$ with a parameter $0<\theta<1$, and showed that its competitive ratio is no less than $1+\frac{\sqrt{10}}{5} \approx 1.632$. For the case $f=2$ and $\theta=\frac{\sqrt{5}-1}{2}$, the authors proved that the algorithm $H_{m}(\theta)$ is best possible with competitive ratio $\frac{\sqrt{5}+1}{2}$. When $m \geqslant 3$ and $\theta=\sqrt{2}-1$, the competitive ratio of algorithm $H_{m}(\theta)$ is no larger than $1+\frac{\sqrt{2}}{2} \approx 1.707$.

A class of above results is listed in Table 1. In the table, if the lower bound is equal to the upper bound of the same problem, we say the problem has a best 
Table 1 For minimizing $C_{\max }$

\begin{tabular}{llll}
\hline Problem & Lower bound & Upper bound & References \\
\hline $1 \mid$ online, p-batch, $b=\infty \mid C_{\max }$ & $\frac{\sqrt{5}+1}{2}$ & $\frac{\sqrt{5}+1}{2}$ & {$[9,35,51]$} \\
$1 \mid$ online, p-batch, $b<\infty($ or $b=2) \mid C_{\max }$ & $\frac{\sqrt{5}+1}{2}$ & 2 (or 1.75) & {$[36,51]$} \\
$1 \mid$ online, p-batch, $b=\infty$, restart $\mid C_{\max }$ & $\frac{5-\sqrt{5}}{2}$ & $\frac{5-\sqrt{5}}{2}$ & {$[14,49]$} \\
$1 \mid$ online, p-batch, $b=\infty, L$-restart $\mid C_{\max }$ & 1.5 & 1.5 & {$[15]$} \\
$1 \mid$ online, p-batch, $p_{j}=p, b=2, L$-restart $\mid C_{\max }$ & $1+\alpha$ & $1+\alpha$ & {$[29]$} \\
$1 \mid$ online, p-batch, $p_{j}=p, b \geqslant 3, L$-restart $\mid C_{\max }$ & $1+\beta$ & $1+\beta$ & {$[29]$} \\
$1 \mid$ online, p-batch, $p_{j}=p, b=2, \operatorname{restart} \mid C_{\max }$ & $1+\alpha$ & $1+\alpha$ & {$[29]$} \\
$1 \mid$ online, p-batch, $p_{j}=p, b=3, \operatorname{restart} \mid C_{\max }$ & $1+\gamma$ & $1+\gamma$ & {$[29]$} \\
$1 \mid$ online, p-batch, $p_{j}=p, b \geqslant 4, \operatorname{restart} \mid C_{\max }$ & $1+\varphi$ & $1+\varphi$ & {$[29]$} \\
$1 \mid$ online, p-batch, $b=\infty, 2$ families $\mid C_{\max }$ & $\frac{\sqrt{17}+3}{4}$ & $\frac{\sqrt{17}+3}{4}$ & {$[16]$} \\
$1 \mid$ online, p-batch, $b=\infty, f$ families $\mid C_{\max }$ & $1+\alpha_{f}$ & $1+\alpha_{f}$ & {$[13]$} \\
$1 \mid$ online, p-batch, $b=\infty, J^{*}(t)\left(\right.$ or $\left.p^{*}(t)\right) \mid C_{\max }$ & $\frac{5-\sqrt{5}}{2}$ & $\frac{5-\sqrt{5}}{2}$ & {$[50]$} \\
$1 \mid$ online, p-batch, $b=\infty, r^{*}(t) \mid C_{\max }$ & 1.442 & 1.5 & {$[50]$} \\
$P 2 \mid$ online, p-batch, $b=\infty \mid C_{\max }$ & $\sqrt{2}$ & $\sqrt{2}$ & {$[33,41]$} \\
$P 2 \mid$ online, p-batch, $L$-restart, $b=\infty \mid C_{\max }$ & 1.298 & $\frac{\sqrt{3}+1}{2}$ & {$[12]$} \\
$P m \mid$ online, p-batch, $p_{j}=p, b=\infty \mid C_{\max }$ & $1+\beta_{m}$ & $1+\beta_{m}$ & {$[52]$} \\
$P m \mid$ online, p-batch, $p_{j}=p, b<\infty \mid C_{\max }$ & $\frac{\sqrt{5}+1}{2}$ & $\frac{\sqrt{5}+1}{2}$ & {$[52]$} \\
$P m \mid$ online, p-batch, $b=\infty \mid C_{\max }$ & $1+\gamma_{m}$ & $1+\gamma_{m}$ & {$[27,40]$} \\
$P m \mid$ online, p-batch, $m$ families, $b=\infty \mid C_{\max }$ & $\frac{\sqrt{5}+1}{2}$ & $1+\frac{\sqrt{2}}{2}$ & {$[42]$} \\
\hline & & & \\
\hline
\end{tabular}

possible online algorithm and it has been solved thoroughly. It leaves the status of the problem open if there exists a gap between the lower bound and the upper bound. Two additional notations will appear in the table. Let $\beta_{m}$ be the positive solution of equation $(1+\beta)^{m+1}=\beta+2$. Let $\alpha_{f}$ be the positive solution of equation $f \cdot \alpha^{2}+\alpha-f=0$, i.e., $\alpha_{f}=1+\frac{\sqrt{4 f^{2}+1}-1}{2 f}$. Let $\gamma_{m}$ be the positive solution of equation $\gamma^{2}+m \gamma-1=0$, i.e., $\gamma_{m}=\frac{\sqrt{m^{2}+4}-m}{2}$. Let $\alpha \approx 0.29, \beta \approx 0.47, \gamma \approx 0.38$, and $\varphi \approx 0.40$ be the positive solution of $(1+\alpha)\left(2 \alpha^{2}+4 \alpha+1\right)=3, \beta(1+\beta)^{2}=1$, $\gamma(\gamma+1)(2 \gamma+3)=2$, and $\varphi(\varphi+1)(2 \varphi+1)=1$, respectively.

We present the following open problems for further research:

(1) $P m \mid$ online, p-batch, $f$ families, $b=\infty \mid C_{\max }$.

(2) $P m \mid$ online, p-batch, restart, $b=\infty \mid C_{\max }$.

(3) $P m \mid$ online, p-batch, L-restart, $b=\infty \mid C_{\max }$.

(4) $P m \mid$ online, p-batch, $J^{*}(t), b=\infty \mid C_{\max }$.

(5) $P m \mid$ online, p-batch, $p^{*}(t), b=\infty \mid C_{\max }$.

(6) $P m \mid$ online, p-batch, $r^{*}(t), b=\infty \mid C_{\max }$.

Furthermore, for problem $1 \mid$ online, p-batch, $b<\infty \mid C_{\max }$, the known lower bound is 1.618 and the known upper bound is 2. Poon and $\mathrm{Yu}$ [36] presented an online 
algorithm of competitive ratio $7 / 4$ when $b=2$. For general $b$, it remains a long standing and challenging open problem.

\section{To Minimize the Maximum Delivery Time $D_{\max }$}

Many researchers studied online scheduling problems with delivery times. The objective is to minimize the time by which all jobs have been delivered, denoted by $D_{\max }$. Hoogeveen and Vestjens [19] first studied online problem on a single machine with delivery time, and they presented a best possible online algorithm with competitive ratio $\frac{\sqrt{5}+1}{2}$. Recently, Tian et al. first investigated an online model with delivery time on a parallel-batch machine. Tian et al. [39] proposed three online algorithms to solve the problem on a single batch machine to minimize the maximum delivery time. The lower bound $\frac{\sqrt{5}+1}{2}$ presented by Zhang et al. [51] is also appropriate for this model. Tian et al. [39] provided a 2-competitive algorithm for $b=\infty$ and a 3-competitive algorithm for $b<\infty$. When the jobs have equal processing time, they present best possible algorithms for arbitrary batch capacity $b$. Under unbounded setting, if the processing time $p_{j}$ and the delivery time $q_{j}$ of every job $J_{j}$ satisfy some restricted properties, Yuan et al. [48], Tian et al. [43], and Fang et al. [10] proposed best possible online algorithms with competitive ratio $\frac{\sqrt{5}+1}{2}$. Later, for general case, Tian et al. [44] improved the competitive ratio 2 to $2 \sqrt{2}-1$. It still leaves a gap between the lower bound and the upper bound. Fang et al. [10] studied the problem on $m$ parallel-batch machines. They used the lower bound $1+\alpha_{m}$ where $\alpha_{m}^{2}+m \alpha_{m}=1$, which was presented by Liu et al. [27] and Tian et al. [40]. Fang et al. [11] designed an online algorithm with competitive ratio $1.5+o(1)$ (no greater than 2), and then Liu et al. [28] presented a new algorithm improving the

Table 2 For minimizing $D_{\max }$

\begin{tabular}{llll}
\hline Problem & Lower bound & Upper bound & References \\
\hline $1 \mid$ online, p-batch, $b=\infty, q_{j} \mid D_{\max }$ & $\frac{\sqrt{5}+1}{2}$ & 2 & [39] \\
$1 \mid$ online, p-batch, $b<n, q_{j} \mid D_{\max }$ & $\frac{\sqrt{5}+1}{2}$ & 3 & [39] \\
$1 \mid$ online, p-batch, $b, p_{j}=p, q_{j} \mid D_{\max }$ & $\frac{\sqrt{5}+1}{2}$ & $\frac{\sqrt{5}+1}{2}$ & [39] \\
$1 \mid$ online, p-batch, $b=\infty, q_{j} \mid D_{\max }$ & $\frac{\sqrt{5}+1}{2}$ & $2 \sqrt{2}-1$ & [44] \\
$1 \mid$ online, p-batch, $b=\infty, \operatorname{Agreeable}\left(p_{j}, q_{j}\right) \mid D_{\max }$ & $\frac{\sqrt{5}+1}{2}$ & $\frac{\sqrt{5}+1}{2}$ & [48] \\
$1 \mid$ online, p-batch, $b=\infty, p_{j} \geqslant q_{j} \mid D_{\max }$ & $\frac{\sqrt{5}+1}{2}$ & $\frac{\sqrt{5}+1}{2}$ & [48] \\
$1 \mid$ online, p-batch, $b=\infty, p_{j} \leqslant q_{j} \mid D_{\max }$ & $\frac{\sqrt{5}+1}{2}$ & $\frac{\sqrt{5}+1}{2}$ & [43] \\
$1 \mid$ online, p-batch, $b=\infty, p_{j} \in\left[p, \frac{\sqrt{5}+1}{2} p\right] \mid D_{\max }$ & $\frac{\sqrt{5}+1}{2}$ & $\frac{\sqrt{5}+1}{2}$ & [10] \\
Pm $\mid$ online, p-batch, $b=\infty, p_{j} \mid D_{\max }$ & $1+\alpha_{m}$ & $1.5+o(1)$ & [11] \\
Pm $\mid$ online, p-batch, $b=\infty, p_{j} \mid D_{\max }$ & $1+\alpha_{m}$ & $1+\frac{2}{\lfloor\sqrt{m}]}$ & [28] \\
Pm $\mid$ online, p-batch, $b=\infty, \operatorname{Agreeable}\left(p_{j}, q_{j}\right) \mid D_{\max }$ & $1+\alpha_{m}$ & $1+\alpha_{m}$ & [28] \\
\hline
\end{tabular}


upper bound to $1+\frac{2}{\lfloor\sqrt{m}\rfloor}$. The latter authors also proposed a best possible online algorithm for a restricted version.

The main results of online scheduling on parallel-batch machines with delivery time are summarized in Table 2.

In this part, we present two open problems for future research.

(1) $1 \mid$ online, p-batch, $b=\infty, q_{j} \mid D_{\max }$.

(2) $\quad P m \mid$ online, p-batch, $b=\infty, q_{j} \mid D_{\max }$.

\section{Other Results}

Li and Yuan [24] studied the online scheduling on $m$ unbounded parallel-batch machines to minimize maximum flow time. They claimed that no online algorithm has a competitive ratio less than $1+\lambda_{m}$, where $\lambda_{m}^{2}+(m+1) \lambda_{m}=1$. An online algorithm with a competitive ratio of $1+\frac{1}{m}$ was proposed. When all jobs have equal processing time, they designed a best possible algorithm with a competitive ratio of $1+\lambda_{m}$.

Li et al. [23] investigated incompatible families on multiple batch machines where each batch has a capacity $b$ with $b=\infty$ under unbounded setting and $b<\infty$ under bounded setting. The objective function is to maximize the weighted number of early jobs. They assumed that the processing time of each job $J$ is equal-length integer, and the release time and the deadline are integer too. When the processing time is of unit length, they first established a lower bound $2-\frac{1}{b}$ and then provided a greedy online algorithm with a competitive ratio of 2 . Then, the algorithm is best possible when $b=\infty$. If the processing time is an arbitrary integer, they presented a $(3+2 \sqrt{2})$-competitive algorithm for both $b=\infty$ and $b<\infty$, which is the first algorithm with constant competitive ratio for this problem.

To minimize the total weighted completion times of jobs on a single batch machine under online setting, for the unbounded case, Chen et al. [7] provided a linear-time online algorithm with a competitive ratio of $\frac{10}{3}$ and a randomized online algorithm with a competitive ratio of 2.89. For the bounded model, they gave an algorithm with a competitive ratio of $4+\epsilon$ and a randomized online algorithm with competitive ratio of $2.89+\epsilon$ for any $\epsilon>0$.

Acknowledgments The authors would like to thank the anonymous referee for his/her constructive comments and kind suggestions.

\section{References}

[1] Anderson, E.J., Potts, C.N.: Online scheduling of a single machine to minimize total weighted completion time. Math. Oper. Res. 29, 686-697 (2004) 
[2] Avramidis, A.N., Healy, K.J., Uzsoy, R.: Control of a batch processing machine: a computational approach. Int. J. Prod. Res. 36, 3167-3181 (1998)

[3] Averbakh, I., Baysan, M.: Batching and delivery in semi-online distribution systems. Discret. Appl. Math. 161, 28-42 (2013)

[4] Brucker, P., Gladky, A., Hoogeveen, H., Kovalyov, M.Y., Potts, C.N., Tautenhahn, T., van de Velde, S.L.: Scheduling a batching machine. J. Sched. 1, 31-54 (1998)

[5] Brucker, P.: Scheduling Algorithms. Springer, Berlin (2003)

[6] Brucker, P., Knust, S.: Complexity results for scheduling problems, http://www.mathematik. uniosnabrueck.de/research/OR/class/ (2007)

[7] Chen, B., Deng, X.T., Zang, W.: On-line scheduling a batch processing system to minimize total weighted job completion time. J. Comb. Optim. 8, 85-95 (2004)

[8] Cheng, T.C.E., Kellerer, H., Kotov, V.: Semi-on-line multiprocessor scheduling with given total processing time. Theor. Comput. Sci. 337, 134-146 (2005)

[9] Deng, X.T., Poon, C.K., Zhang, Y.Z.: Approximation algorithms in batch processing. J. Comb. Optim. 7, 247-257 (2003)

[10] Fang, Y., Liu, P.H., Lu, X.W.: Optimal on-line algorithms for one batch machine with grouped processing times. J. Comb. Optim. 22, 509-516 (2011)

[11] Fang, Y., Lu, X.W., Liu, P.H.: Online batch scheduling on parallel machines with delivery times. Theor. Comput. Sci. 412, 5333-5339 (2011)

[12] Fu, R.Y., Cheng, T.C.E., Ng, C.T., Yuan, J.J.: Online scheduling on two parallel-batching machines with limited restarts to minimize the makespan. Inf. Process. Lett. 110, 444-450 (2010)

[13] Fu, R.Y., Cheng, T.C.E., Ng, C.T., Yuan, J.J.: A best online algorithm for a single parallel batch machine scheduling with $f$ job families. Opera. Res. Lett. 41, 216-219 (2013)

[14] Fu, R.Y., Tian, J., Yuan, J.J., Lin, Y.X.: On-line scheduling in a parallel batch processing system to minimize makespan using restarts. Theor. Comput. Sci. 374, 196-202 (2007)

[15] Fu, R.Y., Tian, J., Yuan, J.J., He, C.: On-line scheduling on a batch machine to minimize makespan with limited restarts. Oper. Res. Lett. 36, 255-258 (2008)

[16] Fu, R.Y., Tian, J., Yuan, J.J.: On-line scheduling on an unbounded batch machine to minimize makespan of two families of jobs. J. Sched. 12, 91-97 (2009)

[17] Hall, N.G., Posner, M.E., Potts, C.N.: Online scheduling with known arrival times. Math. Oper. Res. 34, 92-102 (2009)

[18] Hoogeveen, J.A., Vestjens, A.P.A.: Optimal on-line algorithms for single-machine scheduling. Lect. Notes Comput. Sci. 1996, 404-414 (1084)

[19] Hoogeveen, J.A., Vestjens, A.P.A.: A best possible deterministic on-line algorithm for minimizing maximum delivery time on a single machine. SIAM J. Discret. Math. 13, 56-63 (2000)

[20] Lawler, E.L., Lenstra, J.K., Rinnooy Kan, A.H.G., Shmoys, D.B.: Sequencing and scheduling: algorithms and complexity, in Logistics of Production and Inventory. In: Graves, S.C., Zipkin, P.H., Rinnooy Kan, A.H.G. (eds.) Handbooks Operation Research Management Science, pp. 445-522. North-Holland, Amsterdam (1993)

[21] Lee, C.Y., Uzsoy, R., Martin-Vega, L.A.: Efficient algorithms for scheduling semiconductor burn-in operations. Oper. Res. 40, 764-775 (1992)

[22] Lee, C.Y., Uzsoy, R.: Minimizing makespan on a single batch processing machine with dynamic job arrivals. I. J. Prod. Res. 37, 219-236 (1999)

[23] Li, W.J., Zhang, Z.K., Liu, H.L., Yuan, J.J.: Online scheduling of equal-length jobs with incompatible families on multiple batch machines to maximize the weighted number of early jobs. Inf. Process. Lett. 112, 503-508 (2012)

[24] Li, W.H., Yuan, J.J.: Online scheduling on unbounded parallel-batch machines to minimize maximum flow-time. Inf. Process. Lett. 111, 907-911 (2011)

[25] Li, W.H., Zhang, Z.K., Yang, S.F.: Online algorithms for scheduling unit length jobs on parallelbatch machines with lookahead. Inf. Process. Lett. 112, 292-297 (2012)

[26] Liu, Z.H., Yu, W.: Scheduling one batch processor subject to job release date. Discret. Appl. Math. 105, 129-136 (2000)

[27] Liu, P.H., Lu, X.W., Fang, Y.: A best possible deterministic on-line algorithm for minimizing makespan on parallel batch machines. J. sched. 15, 77-81 (2012)

[28] Liu, P.H., Lu, X.W.: Online unbounded batch scheduling on parallel machines with delivery times. Journal of Combinatorial Optimization (2014). doi:10.1007/s10878-014-9706-4

[29] Liu, H.L., Yuan, J.J.: Online scheduling of equal length jobs on a bounded parallel batch machine with restart or limited restart. Theor. Comput. Sci. 543, 24-36 (2014) 
[30] Ma, R., Wan, L., Wei, L.J., Yuan, J.J.: Online bounded-batch scheduling to minimize total weighted completion time on parallel machines. Int. J. Prod. Econ. 156, 31-38 (2014)

[31] Mathirajan, M., Sivakumar, A.I.: A literature review, classification and simple meta-analysis on scheduling of batch processors in semiconductor. Int. J. Adv. Manuf. Technol. 29, 990-1001 (2006)

[32] Nong, Q.Q., Yuan, J.J., Fu, R.Y., Lin, L., Tian, J.: The single-machine parallel-batching on-line scheduling problem with family jobs to minimize makespan. Intern. J. Prod. Econ. 111, 435-440 (2008)

[33] Nong, Q.Q., Cheng, T.C.E., Ng, C.T.: An improved on-line algorithm for scheduling on two unrestrictive parallel batch processing machines. Oper. Res. Lett. 36, 584-588 (2008)

[34] Poon, C.K., Zhang, P.X.: Minimizing makespan in batch machine scheduling. Algorithmica 39, 155-174 (2004)

[35] Poon, C.K., Yu, W.C.: A flexible online scheduling algorithm for batch machine with infinite capacity. Ann. Oper. Res. 133, 175-181 (2005)

[36] Poon, C.K., Yu, W.C.: On-line scheduling algorithms for a batch machine with finite capacity. J. Comb. Optim. 9, 167-186 (2005)

[37] Seiden, S., Sgall, J., Woeginger, G.: Semi-online scheduling with decreasing job sizes. Oper. Res. Lett. 27, 215-221 (2000)

[38] Tan, Z.Y., He, Y.: Semi-on-line problems on two identical machines with combined partial information. Oper. Res. Lett. 30, 408-414 (2002)

[39] Tian, J., Fu, R.Y., Yuan, J.J.: On-line scheduling with delivery time on a single batch machine. Theor. Comput. Sci. 374, 49-57 (2007)

[40] Tian, J., Cheng, T.C.E., Ng, C.T., Yuan, J.J.: Online scheduling on unbounded parallel-batch machines to minimize makespan. Inf. Process. Lett. 109, 1211-1215 (2009)

[41] Tian, J., Fu, R.Y., Yuan, J.J.: A best online algorithm for scheduling on two parallel batch machines. Theor. Comput. Sci. 410, 2291-2294 (2009)

[42] Tian, J., Cheng, T.C.E., Ng, C.T., Yuan, J.J.: Online scheduling on unbounded parallel-batch machines with incompatible job families. Theor. Comput. Sci. 412, 2380-2386 (2011)

[43] Tian, J., Fu, R.Y., Yuan, J.J.: An on-line algorithm for the single machine unbounded parallelbatching scheduling with large delivery times. Inf. Process.g Lett. 111, 1048-1053 (2011)

[44] Tian, J., Cheng, T.C.E., Ng, C.T., Yuan, J.J.: An improved on-line algorithm for single parallelbatch machine scheduling with delivery times. Discret. Appl. Math. 160, 1191-1210 (2012)

[45] Uzsoy, R., Lee, C.Y., Martin-Vega, L.A.: A review of production planning and scheduling models in the semiconductor industry, part I: system characteristics, performance evaluation and production planning. IIE Trans. Sched. Logist. 24, 47-61 (1992)

[46] Uzsoy, R., Lee, C.Y., Martin-Vega, L.A.: A survey of production planning and scheduling models in the semiconductor industry, part II: shop-floor control. IIE Trans. Sched. Logist. 26, 44-55 (1994)

[47] Yuan, J.J.: Online over time scheduling on unbounded p-batch machines to minimize the makespan: a survey http://www.paper.edu.cn (2010)

[48] Yuan, J.J., Li, S.S., Tian, J., Fu, R.Y.: A best possible on-line algorithm for the single machine parallel-batch scheduling with restricted delivery times. J. Comb. Optim. 17, 206-213 (2009)

[49] Yuan, J.J., Fu, R.Y., Ng, C.T., Cheng, T.C.E.: A best on-line algorithm for unbounded parallel batch scheduling to minimize makespan with restarts. J. Sched. 14, 361-369 (2011)

[50] Yuan, J.J., Ng, C.T., Cheng, T.C.E.: Best semi-online algorithms for unbounded parallel batch scheduling. Discret. Appl. Math. 159, 838-847 (2011)

[51] Zhang, G.C., Cai, X.Q., Wong, C.K.: Online algorithms for minimizing makespan on batch processing machines. Nav. Res. Logist. 48, 241-258 (2001)

[52] Zhang, G.C., Cai, X.Q., Wong, C.K.: Optimal online algorithms for scheduling on parallel batch processing machines. IIE Trans. 35, 175-181 (2003) 